Supporting Information to Vibrational Energy Redistribution between CH Stretching Modes in Alkyl Chain Monolayers Revealed by Time-Resolved Two-Color Pump-Probe Sum Frequency Spectroscopy

Michael Lackner, Marvin Hille, and Eckart Hasselbrink

Fakultät für Chemie and Center for Nanointegration (CENIDE), Universität DuisburgEssen, 45117 Essen, Germany

DELAY SCANS FOR ALL POLARIZATION/PUMPED MODE COMBINATIONS
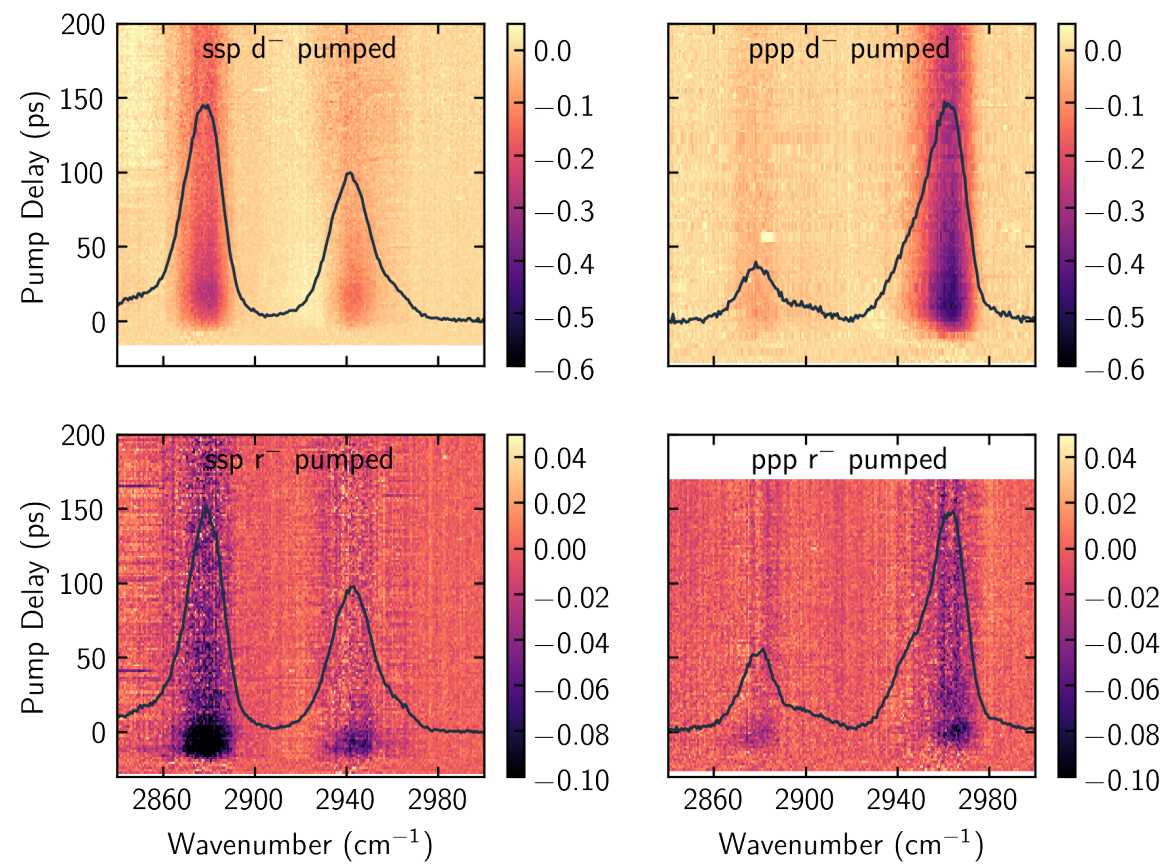

FiguRE S1. Bleach for all four combinations of polarization $(s s p / p p p)$ and pumped mode $\left(d^{-} / r^{-}\right)$. The solid black lines represent the underlying vSF spectra. 


\section{BLEACH LINEARITY}
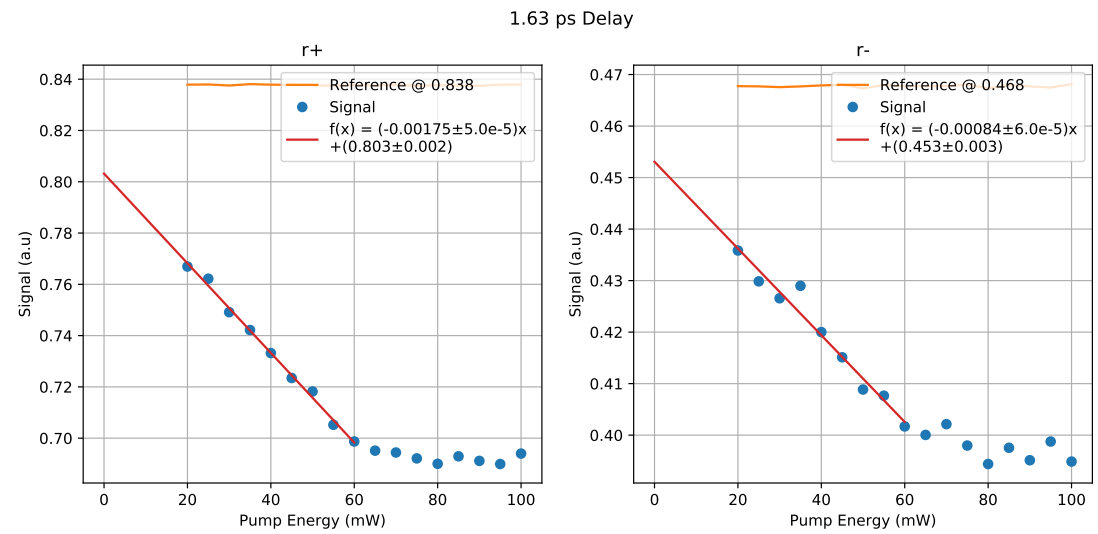

FIGURE S2. The bleach as function of the applied IR pump energy observed on the $r^{-}$and $r^{+}$band. Note, the laser operates at a repetition rate of $1 \mathrm{kHz}$. The data has been recorded for a pump-pulse delay of $1.63 \mathrm{ps}$. 
MOdel Fit PARAMETERS

TABLE S1. Compilation of fit parameters

\begin{tabular}{lr}
\hline Parameter & Value \\
\hline$c_{d}$ & $18 \mathrm{~J} / \mathrm{K}$ \\
$c_{r}$ & $1 \mathrm{~J} / \mathrm{K}$ \\
$c_{b}$ & $60 \mathrm{~J} / \mathrm{K}$ \\
$a_{d r}$ & $0.8 \mathrm{~J} / \mathrm{ps} \mathrm{K}$ \\
$a_{r b}$ & $0.4 \mathrm{~J} / \mathrm{ps} \mathrm{K}$ \\
$a_{d b}$ & $0.4 \mathrm{~J} / \mathrm{ps} \mathrm{K}$ \\
laserwidth & $15 \mathrm{ps}$ \\
$t_{0}$ & $20 \mathrm{ps}$ \\
\hline
\end{tabular}

(1)

$$
\begin{aligned}
\sigma_{\text {Laser }} & =\text { laserwidth } /(2 \sqrt{2 \ln 2}) \\
f_{\text {Laser }}(t) & =\exp \left[-\left(t-t_{0}\right)^{2} / 2 \sigma_{\text {Laser }}^{2}\right] \\
\text { bleach }(t) & \propto\left(1-c_{\text {vib }} *\left(2 * T_{r}(t)+T_{d}(t)\right)\right)^{2}
\end{aligned}
$$




\section{Coherent CONVOLUtion of RESONANCES With OPPOSITE PHASE AND EFFECT OF} $\chi^{(3)}$ ON RECOVERY TIME

The following serves to demonstrate that a "negative" bleach can result from the coherent superposition of two bands that are located within their width and are characterized by opposite phase. Each individual band sees a reduction in oscillator strength by a factor $a_{i}$ :

$$
I_{\mathrm{SF}}=\left|\sum_{i} \frac{A_{i}}{\omega-\omega_{i}-i \Gamma_{i}}\right|^{2}
$$

$$
\Delta I_{\mathrm{SF}}=\left|\sum_{i} \frac{a_{i} A_{i}}{\omega-\omega_{i}-i \Gamma_{i}}\right|^{2}-\left|\sum_{i} \frac{A_{i}}{\omega-\omega_{i}-i \Gamma_{i}}\right|^{2}
$$

For the plots in figure S3 $A_{1}$ and $A_{2}$ were set to +6 and -5 respectively. The negative sign for latter indicates an opposite phase relation between the two resonances. Both $\Gamma_{i}$ were set to $10 \mathrm{~cm}^{-1}, \omega_{1}$ to $2895 \mathrm{~cm}^{-1}$ and $\omega_{2}$ to $2905 \mathrm{~cm}^{-1}$.
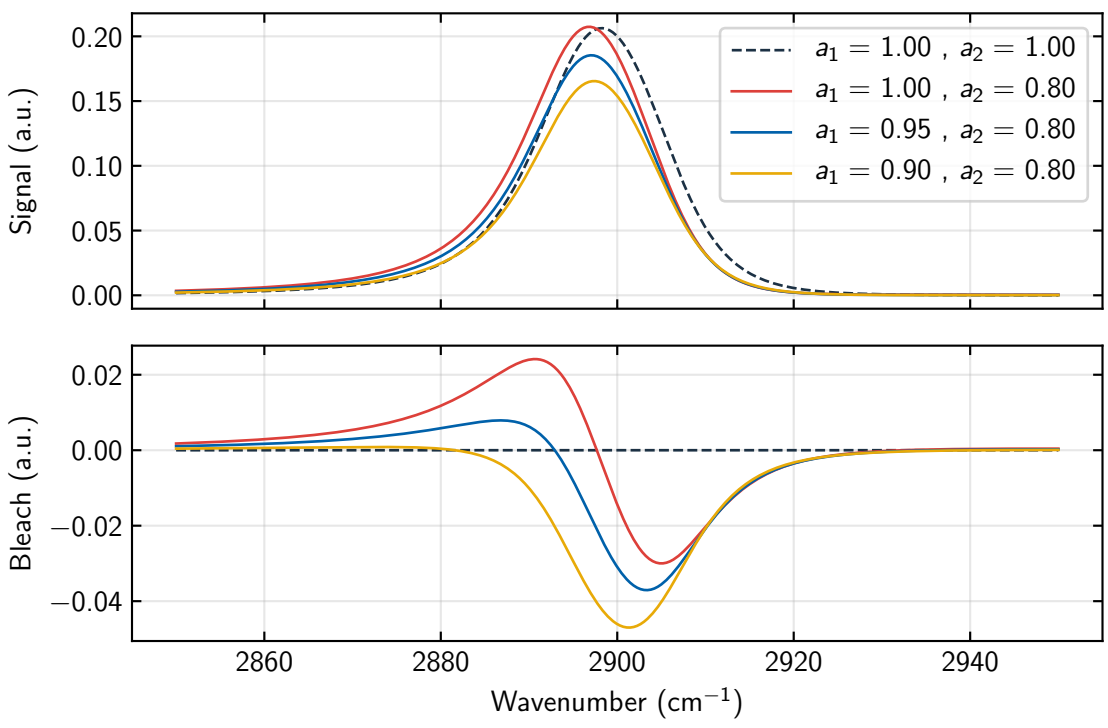

FiguRE S3. Signal and bleach for four exemplary parameters.

We set up a model to evaluate the effect of the likely $\chi^{(3)}$ contribution.

$$
I^{S F S}\left(\omega_{I R}\right) \propto|| \chi^{(3)}\left|e^{i \xi}+\frac{(1-b \exp [-t / \tau])}{\omega_{I R}-\omega+i \Gamma}\right|^{2},
$$


We set $\xi$ to $-\pi, \tau$ to $100 \mathrm{ps}, \Gamma$ to $8 \mathrm{~cm}^{-1}, b$ to 0.9 and $\omega$ to $2880 \mathrm{~cm}^{-1}$. The $\chi^{(3)}$ contribution was set to 0.1 . We then calculated the line shape for $t=\infty$ (s. Fig. 2 left panel). As can been seen the effect of the $\chi^{3}$ contribution is severe and the line shape deviates more from a Lorentzian than anything we observe in the experiment. In order to evaluate the time evolution we calculated the line shapes for times between 0 and $200 \mathrm{ps}$ and integrated as in the experiment over a width of 1.5 times the FWHM, i.e. $24 \mathrm{~cm}^{-1}$, for each time. The result is shown in the right hand panel. Single exponential fits to the result yield recovery time constants of 104.2 and 105.2 ps for the distorted and non-distorted case, respectively. we conclude, that the effect of the $\chi^{(3)}$ contribution on the line shape is large, but rather small on the recovery time derived by our procedure. From the experimental point of view the difference is not resolvable.
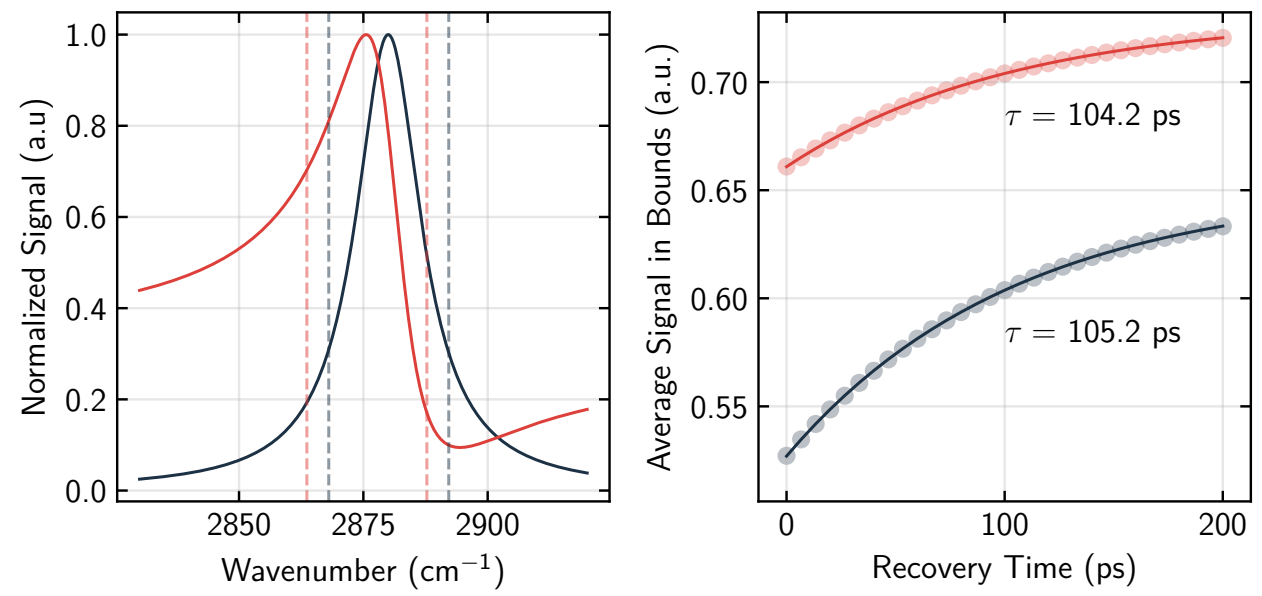

FiguRE S4. left: Line profile without the $\chi^{(3)}$ contribution (blue) and with it (red). right: Temporal evolution of the bleach corresponding to the two scenarios. The tags give the observed recovery times, which only differ by 1 ps and are close to the input value of 100 ps. 
Pump LASER PULSE WIDTh

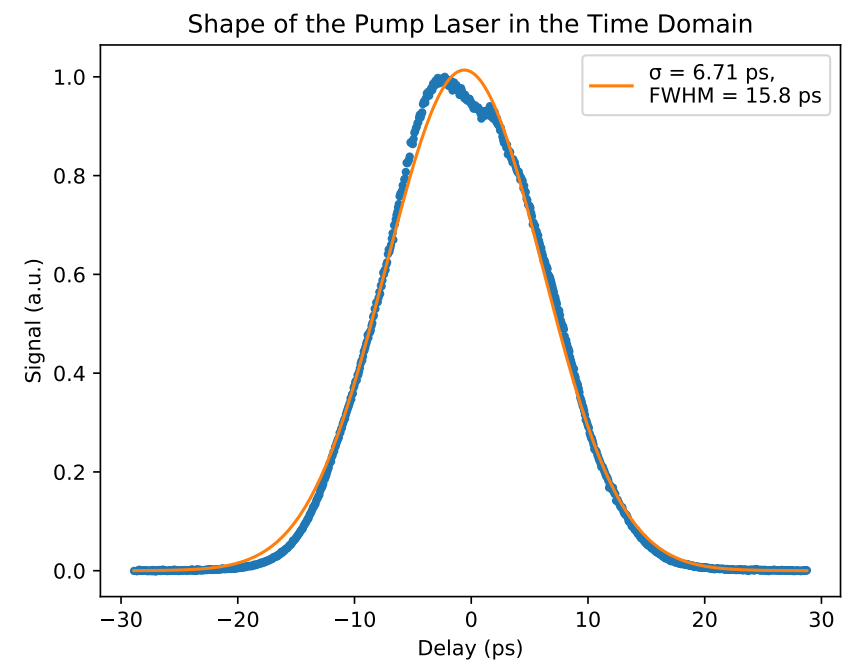

FiguRE S5. Cross correlation ps IR pump laser and $515 \mathrm{~nm}$ upconversion pulse.

Spectral PROfile IR PROBE PULSE

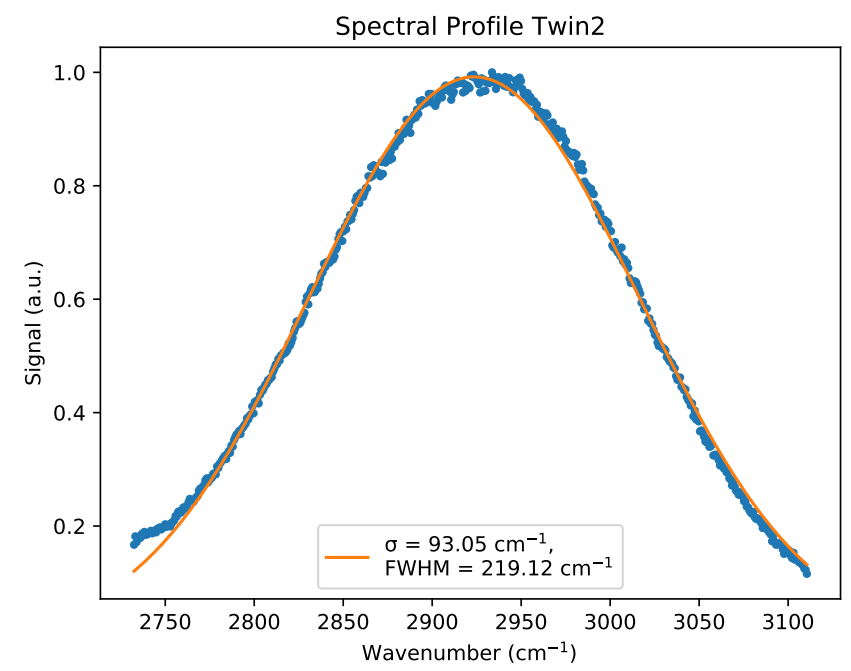

FiguRE S6. Cross correlation fs IR probe laser with $515 \mathrm{~nm}$ upconversion pulse. 